\begin{tabular}{|l|l|l|l|}
\hline Eiszeitalter und Gegenwart & Band 16 & Seite 113-115 & Öhringen/Württ.,31.Dezember 1965 \\
\hline
\end{tabular}

\title{
Über Aufbau und Altersgliederung des Holozäns der Lüneburger Elbmarsch ${ }^{1}$ )
}

\author{
Von Jobst-Henrich Benzler, Hannover
}

Mit 1 Abbildung im Text

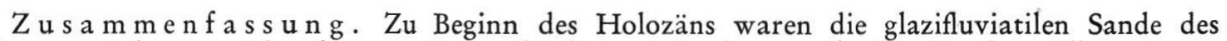
Elburstromtales von Schmelzwasserrinnen durchzogen, in denen teilweise die alten Elbarme verliefen. Diese Sande wurden zu Dünen aufgeweht, auf denen sich Podsole bildeten. Während des gesamten Holozäns lagerte die Elbe an ihren Ufern lehmig-sandige, im Hinterland tonreiche Sedimente $\mathrm{ab}$, die sich mit Torfen in verlandeten Altarmen und im Sietland verzahnen. Die Bedeichung im Mittelalter beendete die allgemeine Sedimentation. Nur bei Deichbrüchen kamen örtlich fächerförmige Sanddecken zur Ablagerung.

Das Alter von Torflagen und Humusdwögen innerhalb der tonigen Sedimente wurde mittels ${ }^{14} \mathrm{C}-$-Untersuchung bestimmt, ebenso von Holzkohle aus den Dünenpodsolen.

$\mathrm{Sum} \mathrm{mary}$. At the beginning of the Holocene the glaciofluvial sands of the Elbe glacial stream valley were traversed by channels of melting waters, through which the old arms of the river Elbe were flowing. The winds accumulated these sands to dunes, on the top of which podzols were formed. During the whole of the Holocene the river Elbe deposited loamy-sandy sediments on the higher zone near the banks, but clayey sediments in the lower zone of the back land. These clayey sediments alternate with peat layers in dead channels of the river Elbe and in the back land. General sedimentation ended through diking during the Middle Ages. Only fan-like sandy layers were deposited locally where dikes were broken.
\end{abstract}

The ages of peat layers and „Humusdwögen“ (humic horizons of fossil soils) between clay sediments, and also of charcoal in the dune podzols were determined by the ${ }^{14} \mathrm{C}$ method.

Seit 1962 führt das Niedersächsische Landesamt für Bodenforschung in Hannover im Rahmen des Küstenplanes geologisch-bodenkundliche Kartierungen in der Lüneburger Elbmarsch zwischen Winsen/Luhe und Bleckede durch. Neben einer Übersichtskartierung des gesamten Gebietes im Maßstab 1:25000 werden bestimmte Bezirke, die für Flurbereinigungs- und Meliorationsmaßnahmen von besonderer Bedeutung sind, im Maßstab $1: 5000$ aufgenommen. Die Bohrtiefe beträgt normalerweise 4 Meter. Im Zusammenhang mit dem Bau der Staustufe Geesthacht erbohrte die hydrogeologische Abteilung des NLfB mehrere Profile bis 10 Meter Tiefe durch die gesamte Lüneburger Elbmarsch. Aus diesen Unterlagen wurde der schematische Schnitt durch die Elbmarsch (Abb.1) zusammengestellt. Die dabei verwendeten Symbole für die einzelnen Folgen der verschiedenen Ablagerungsperioden $\left(\mathrm{m}_{1}-\mathrm{m}_{3}, \mathrm{u}_{1}-\mathrm{u}_{2}, \mathrm{O}_{1}-\mathrm{O}_{4}\right)$ entsprechen den Angaben bei W. MüLLER (1962).

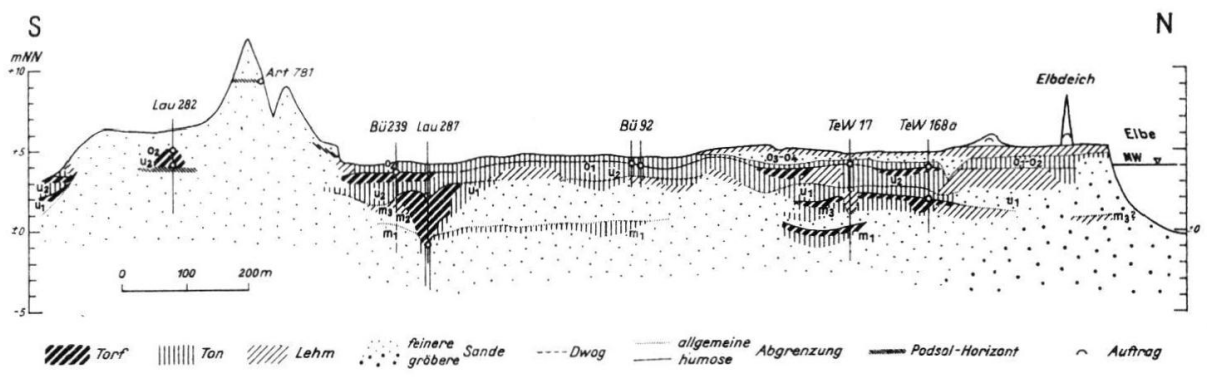

Abb. 1. Schnitt durch die Lüneburger Elbmarsch (schematisch).

1) Vortrag, gehalten auf der 12. Tagung der Deutschen Quartärvereinigung in Lüneburg am 8. 10. 1964.

8 Eiszeit und Gegenwart 
Zu Beginn des Holozäns war das Elbe-Urstromtal zwischen Bleckede und Winsen/Luhe erfüllt von glazifluviatilen Sanden, deren Korngröße von unten nach oben abnahm. Schmelzwasserrinnen zerschnitten das Gebiet, in denen streckenweise die alten Elbarme verliefen. Diese Sande wurden weithin zu Dünen aufgeweht, deren höchste Punkte heute bei etwa $+11 \mathrm{~m}$ NN liegen. Auf diesen Dünen bildeten sich Podsole unterschiedlicher Mächtigkeit und Höhenlage, die später bei Verlagerungen der Dünen teils abgeweht, teils überdeckt wurden und heute meist nur noch in Resten erhalten sind.

Im Altholozän setzte in den Rinnen des Gebietes Verlandung und Vermoorung ein. Die Kalkmudden, die sich am Grund der Rinnen finden, dürften ihren Kalkgehalt vom Geschiebemergel der benachbarten Hochflächen erhalten haben. In Altarmen und im Sietland am Rand der höheren "Vorgeest“ kam es zur Bildung mehrere Meter mächtiger Torfe, vorwiegend Bruchwaldtorfe. Anschließend begann die Sedimentation von meist tonreichem Material (bis $80 \%$ Teilchen unter $2 \mu$ ). Diese Sedimentation wurde verstärkt, als durch den Anstieg des Meeresspiegels der tidebedingte Rückstau des Flusses bis in die Lüneburger Elbmarsch reichte. Die Ablagerungen erfolgten nicht gleichmäßig, sondern in wechselnder Stärke. Dazwischen kam es immer wieder zur Bildung geringmächtiger Torflagen, die das Sediment gliedern. Die Tone enthalten oft größere Mengen organischer Substanz und sind durchweg kalkfrei. Sie stammen wohl überwiegend vom Oberlauf des Flusses und von den benachbarten Hochflächen. $\mathrm{Ob}$ sich auch Material aus dem Tidebereich dabei befindet, das stromauf mit der Tide gewandert sein müßte, läßt sich noch nicht entscheiden. Von Dr. HalliK, Hamburg, und Dr. BEnda, Hannover, durchgeführte Diatomeenanalysen blieben ergebnislos, da die Diatomeen der fraglichen Schichten bis zur Unkenntlichkeit aufgearbeitet und nicht mehr bestimmbar waren.

An der Elbe bildete sich ein Uferwall aus lehmig-sandigem Material, das großenteils vom gegenüberliegenden schleswig-holsteinschen Steilufer stammen dürfte, in das sich die Elbe ständig weiter hineingenagt hat. Zur Karolingerzeit war das gesamte Elbtal von einer Kleidecke überzogen, aus der die Dünen als einzelne Kuppen oder als langgestreckte Rücken herausragten. Die Elbe, die heute durch Deiche und Buhnen in ein festes Bett am Nordrand der Marsch gezwungen wird, zog sich damals in zahlreichen Armen durch das Gebiet, von denen die Wettern, die Ilau und die Unterläufe von Neetze und Ilmenau der Rest sein dürften. Sie waren so flach, daß Karl der Große die Elbe bei Lauenburg auf Furten durchschreiten konnte.

Die allgemeine Sedimentation fand mit der Bedeichung der Elbmarschen im Mittelalter ihr Ende. Bei Deichbrüchen, die bis ins 18. Jahrhundert ständig auftraten, kam es örtlich noch zur Sedimentation. Um die tief ausgekolkten Bracks herum schütteten die eingebrochenen Wassermassen Sandfächer bis zu $2 \mathrm{~km}$ Durchmesser, die im Zentrum mehr als 2 Meter Mächtigkeit aufweisen. Der hohe Anteil nordischen Materials, insbesondere an Feldspäten, und bis zu $3 \mathrm{~mm}$ großen Glimmerplättchen deuten darauf hin, daß diese Sande nicht weit transportiert wurden. Wahrscheinlich stammen auch sie vom gegenüberliegenden Steilufer.

Bei der allgemeinen, durch die vorherrschenden Westwinde bedingten Ostwärtswanderung der Dünen wurden am Osthang der Dünen vielfach tonige Sedimente durch Sandüberwehung überlagert.

In jüngster Zeit wurden die Außendeichsflächen an der Elbe weithin mit Sanden aufgespïlt, die beim Ausbaggern und Begradigen des Flusses anfallen.

Zur Altersgliederung der Sedimente wurden die zwischengeschalteten Torfe und fossilen Bodenbildungshorizonte (Humusdwöge) benutzt, die im NLfB von Herrn GEYH auf ${ }^{14} \mathrm{C}$ untersucht wurden. Die Entnahmepunkte und Tiefen sind in Abb. 1 angegeben.

Die tiefste Rinne, in der heute die Neetze verläuft, reicht bis $4 \mathrm{~m}$ unter NN. In ihr befindet sich ein mehrere Meter mächtiger Torf, dessen Wachstum im Boreal (6470 $180 \mathrm{v}$. 
Chr. Geb., Probe Lau 287) begann und bis ins Subboreal (2110 \pm 100 v. Chr. Geb.) dauerte. Eine weniger mächtige Torflage bei $+2 \mathrm{~m}$ NN gehört ins Ende des Atlantikums

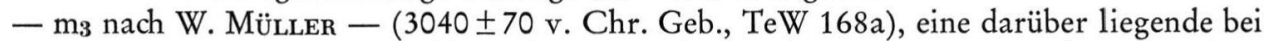

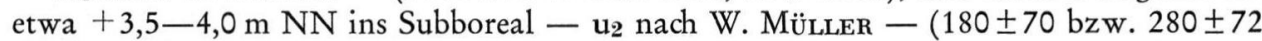
v. Chr. Geb., TeW 168a bzw. Bü 239). Die ungefähr $+4-4,5 \mathrm{~m}$ NN liegenden Humusdwöge sind subatlantisch $-O_{1}$ nach W. Müller $-(550 \pm 70$ bzw. $640 \pm 60$ nach Chr. Geb., TeW 17 bzw. Bü 92), ebenso eine dazugehörige humose Rinnenfüllung (470 \pm 50 nach Chr. Geb., Bü 92).

In einem Tümpel am Dünenfuß dauerte das Torfwachstum nach ${ }^{14} \mathrm{C}$-Untersuchung vom Subboreal (530 \pm 60 v. Chr. Geb., Lau 282) bis ins Subatlantikum (930 50 nach Chr. Geb.).

Holzkohle aus dem Bleichhorizont eines Podsols auf einer Düne (Art 781) hatte ein Alter von $1820 \pm 75$ v. Chr. Geb., ist also bronzezeitlich.

Auf den Dünen, die in der ständig von Hochwasser bedrohten Elbmarsch bis zur Bedeichung die einzig sicheren Siedlungsstellen waren, fanden sich verschiedene vorgeschichtliche Siedlungsplätze. Nach den bisher gefundenen Scherben, die von Dr. NowотнNING, Hannover, und Dr. Köster, Lüneburg, bestimmt wurden, handelt es sich um Siedlungen aus dem Zeitraum von der Bronzezeit bis zum Mittelalter. Nähere Untersuchungen sollen noch erfolgen.

Aus den vorliegenden Daten ergibt sich, daß Sedimentation und Torfbildung in der Lüneburger Elbmarsch während des gesamten Holozäns erfolgten, wobei bald die eine, bald die andere vorherrschte, wie aus der Wechsellagerung von Ton und Torf hervorgeht. Die Altersangaben zeigen ferner, daß sich diese verschiedenen Ablagerungsfolgen in das für die küstennahen Marschengebiete aufgestellte Schema einordnen lassen, obwohl wir uns hier am äußersten Rande des Tidebereiches befinden. Die Korrelierung einzelner Schichten ist aber hier viel schwieriger als in Küstennähe. Durch die häufige Verlagerung der alten Elbarme ist die ursprüngliche Schichtung weithin zerstört. Der starke Wechsel der Schichten in horizontaler und vertikaler Richtung sowie die sehr unterschiedliche Setzung infolge sehr unterschiedlicher Bodenarten an den verschiedenen Stellen erschwert die Zuordnung einzelner Horizonte über größere Entfernungen hinweg. Außerdem steigt die Geländeroberfläche der Elbmarsch von Winsen bis Bleckede von etwa $+2 \mathrm{~m}$ auf $+8 \mathrm{~m} \mathrm{NN}$ an. Eine sichere Korrelierung einzelner Schichten ist daher oft nur bei einer Feinkartierung möglich.

\section{Literaturverzeichnis}

Benzler, J.-H.: Beihefte zu Blatt Tespe-West und Bütlingen der Geologisch-bodenkundlichen Karte der niedersächsischen Marschen 1:5000, herausgegeben vom Niedersächsischen Landesamt für Bodenforschung, Hannover 1964. - - Bodenkundliche Karte der Marschengebiete $1: 25000$, Blatt Artlenburg und Lauenburg, herausgegeben vom NLfB, Hannover (im Druck).

MüLleR, W.: Der Ablauf der holozänen Meerestransgression der südlichen Nordseeküste und Folgerungen in bezug auf eine geochronologische Holozängliederung. - Eiszeitalter und Gegenwart 13, S. 197-226, 1962.

Manuskr. eingeg. 2. 3.65.

Anschrift des Verf.: Dr. J.-H. Benzler, Niedersächsisches Landesamt für Bodenforschung, 3 Hannover-Buchholz, Alfred-Bentz-Haus. 